\section{Walking in a Winter Wonderland}

World Wide Web is the most useful tool for finding information, and great fun to play with in your free time. It is delightfully simple to use: when the Mosaic viewer program is started, a 'home page' appears. Simply click on a highlighted phrase that interests you and the relevant document appears on your screen. This document can contain text, pictures, moving images and sounds, and more importantly, new highlighted phrases pointing to yet more documents. Topics ranging from paintings at Le WebLouvre to preprints of scientific papers are covered.

The World Wide Web originated at CERN in 1990, where it was created for physicists to share information within complex, distributed teams. Three years later, it hit the big time when the Mosaic software was developed. The World Wide Web is the fastest growing service on the Internet, which is itself expanding at an incredible rate. "World Wide Web drives the explosion of the global internet," says Robert Cailliau, of CERN's WWW Support Service.

Now, you too can experience the hubbub by using the Web. As all CERN computers have an Internet connection, it is straightforward. Mosaic is usually already installed on CERN's UNIX workstations; just type XMosaic. For Macintoshes, drag Mosaic onto your hard disk from the WWW Support server on Appletalk Zone LDI_2; for PCs, the software is in Communications.

Or you can add to the commotion by publishing information on your experiment, project, group or division. CERN's WWW Support Service will provide full text indexing, cross linking, image capture, back up, access protection, and ultimately statistics on the popularity of your material. All you need do is e-mail them at www-support@www.cern.ch.

\section{Oh! le Waste Wéseau que Woilà}

World Wide Web (littéralement le tissu mondial) est le plus utile des outils pour trouver les informations et une grande source d'amusement dans votre temps libre. Son utilisation est merveilleusement simple:lorsqueleprogrammed'affichage Mosaic est lancé, une paged'aiguillage("home page") apparaît. Cliquez simplement sur l'un des mots saillants qui vous intéresse et le document en question apparaît sur votre écran. Ce document peut contenir un texte, des images fixes ou mobiles et des sons et, le plus important, de nouveaux mots saillants annonçant encore d'autres documents. Les sujets traités vont des tableaux, sous Le WebLouvre, aux pré-tirages $\mathrm{d}^{\prime}$ articles scientifiques.

Le service WWW a pris son origine au CERN en 1990 où il a été créé pour des physiciens afin de partager les informations au sein d'équipes compliquées et dispersées. Trois années plus tard il est devenu célèbre grâce à la mise au point du logiciel Mosaic. Sur le réseau Internet qui lui-même se développe à une vitesse incroyable, World Wide Web a la croissance la plus rapide. "C'est World Wide Web qui sous-tend l'explosion planétaire d'Internet" déclare Robert Cailliau du Service de soutien CERN à WWW.

Maintenant vous pouvez aussi plonger dans le vacarme en utilisant "le Web". Comme tous les ordinateurs du CERN sont connectés à Internet, c' est facile. Normalement Mosaic est déjà installé sur les postes de travail Unix du CERN, tapez simplement XMosaic. Avec les Macintosh, prenez Mosaic sur le serveur WWW Support dans la zone Appletalk LDI-2 et tirez-le jusqu'à votre disque dur; sur les PC le logiciel se trouve dans Communications.

Vous pouvez mêler votre voix au brouhaha en publiant des documents sur votre expérience, projet, groupe ou division. Le service de soutien à WWW du CERN se chargera de l'indexation complète des textes, des références croisées, de la saisie d'images, de la sauvegarde, de la protection de l'accès et enfin des statistiques sur la popularité de votre information. Il vous suffit de joindre le service par courrier électronique à www-support@www.cern.ch.

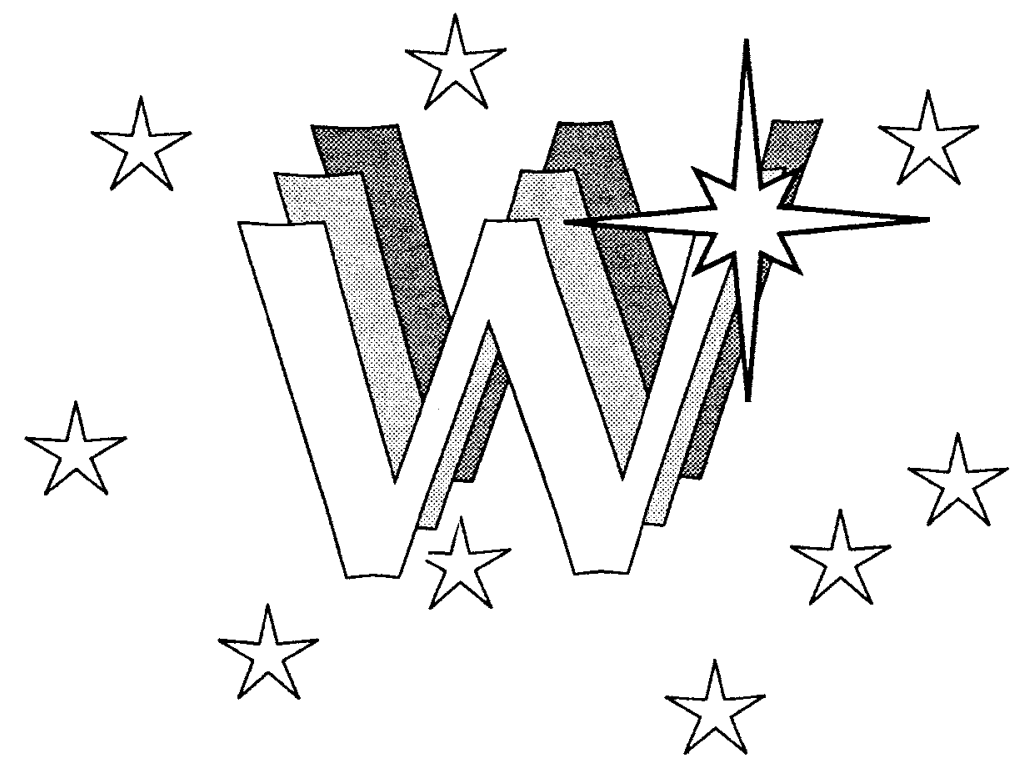

